\title{
Association between Novel Marker (Platelet-Lymphocyte Ratio, Neutrophil-Lymphocyte Ratio, and Delta Neutrophil Index) and Outcomes in Sudden Cardiac Arrest Patients
}

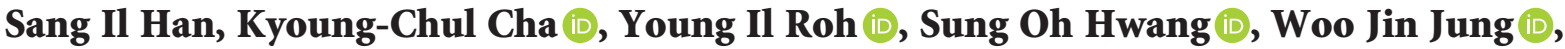 \\ and Tae Youn Kim (D)
}

Department of Emergency Medicine, Yonsei University Wonju College of Medicine, Wonju, Republic of Korea

Correspondence should be addressed to Tae Youn Kim; emkty@yonsei.ac.kr

Received 21 December 2020; Revised 12 March 2021; Accepted 17 March 2021; Published 24 March 2021

Academic Editor: Bartlomiej Perek

Copyright (c) 2021 Sang Il Han et al. This is an open access article distributed under the Creative Commons Attribution License, which permits unrestricted use, distribution, and reproduction in any medium, provided the original work is properly cited.

\begin{abstract}
Purpose. It is important that clinicians accurately predict the outcome of patients with sudden cardiac arrest (SCA). The complete blood count (CBC) is an easy and inexpensive test that provides information on blood content. Platelet-lymphocyte ratio (PLR), neutrophil-lymphocyte ratio (NLR), and delta neutrophil index (DNI) are relatively novel biomarkers that have been used in the prognosis of various diseases. We aimed to determine the usefulness of PLR, NLR, and DNI in predicting the outcomes of SCA. Materials and Methods. This retrospective observational study was performed on patients with SCA. Patients who visited the tertiary university hospital from January 2015 to December 2019 were targeted. The inclusion criteria were all nontraumatic adult out-hospital cardiac arrest patients. We analyzed DNI, PLR, and NLR based on the CBC results of all enrolled patients. The exclusion criteria were as follows: no data on laboratory study, traumatic arrest, age $<18$ years, and a history of leukemia, myelodysplastic syndrome, and myelofibrosis. The primary outcome was assessed as return of spontaneous circulation (ROSC), the secondary outcome as survival to discharge, and the tertiary outcome as neurological outcome. Results. From January 1, 2015, to December 31, 2019, 739 patients were enrolled. ROSC was seen in 324 patients, of whom 60 had survival to discharge and 24 had good neurological outcome at the time of discharge (cerebral performance categories (CPCs) 1-2). The PLR of the ROSC group was 42.41 (range: $4.21-508.7$ ), which was higher than that of the No-ROSC group $(p=0.006)$. The DNI value of the survival group was 0.00 (range: 0.00-40.9), which was lower than that of the nonsurvival group. Conclusions. Patients with SCA and subsequent ROSC had higher PLR and NLR, while those with survival to discharge had lower DNI values than those with nonsurvival to discharge $(p=0.005)$.
\end{abstract}

\section{Introduction}

Outcome assessment after acute cardiac arrest is important to determine treatment regimen [1]. Currently, $80 \%$ of sudden cardiac arrests (SCAs) have a cardiac origin, such as heart failure or acute coronary syndrome including myocardial infarction. The rest are of noncardiac origin, including respiratory problems, cerebrovascular problems, trauma, and metabolic disorders [2]. In accordance with the advanced cardiopulmonary life support (ACLS) algorithm [3], when cardiac arrest occurs, laboratory study or imaging is conducted to identify reversible causes [4]. Based on laboratory studies, the following $\mathrm{Hs}$ and Ts should be treated: hypovolemia, hypoxia, hydrogen ions, and hypo/ hyperkalemia [5]. Therefore, laboratory studies are essential for patients with cardiac arrest, and tests that can predict prognosis through serial follow-up have been reported [6-8].

The complete blood count (CBC) is an easy, inexpensive, and routine test that is often used in emergency rooms to provide information about blood content. It can determine white blood cell count, red blood cell count, platelet count, neutrophil count, lymphocyte count, and monocyte count. Neutrophil-to-lymphocyte ratio (NLR) and platelet to lymphocyte ratio (PLR) are used to predict prognosis in various diseases [9-11]. 
In particular, PLR is associated with malignant tumors, cardiovascular disease, and heart failure [10], probably because inflammatory processes and excessive thrombus activity influence pathogenesis in these diseases [12]. High NLR is associated with most cardiovascular disease outcomes, including acute coronary syndrome, stroke, and composite cardiovascular events [13]. The delta neutrophil index (DNI) has been studied by several research groups as a serum biomarker in infectious and inflammatory conditions [14]. It has implications for sepsis severity and may be valuable to assess prognosis in patients with suspected sepsis [15].

Postcardiac arrest syndrome denotes the biological and clinical manifestations of ischemia-reperfusion injuries triggered by cardiac arrest and return of spontaneous circulation (ROSC) [16]. Systemic ischemia/reperfusion due to cardiac arrest and ROSC is characterized by the release of systemic inflammatory cytokines and generalized activation of leukocytes and endothelial cells [17]. When cardiac arrest occurs, immature neutrophils enter circulation. The elevated immature/total granulocyte ratio is referred to as a left shift [15]. Ischemic damage due to cardiac arrest is associated with more severe apoptosis of T cells and may lead to lower lymphocyte counts [18]. Anticoagulant protein C is consumed rapidly within 2 hours after an arrest, and a short hypocoagulant condition is replaced by a prolonged hypercoagulant condition that causes microcirculatory obstruction, severe lactic acidosis, thrombocytosis, and progressive multiorgan failure [19]. The severity of such disorders varies based on the severity of the ischemic insult, the cause of cardiac arrest, and the patient's state of health before the cardiac arrest [20].

We conducted this study to investigate the significance of PLR, NLR, and DNI in the assessment of outcomes after cardiac arrest.

\section{Methods}

2.1. Study Design and Setting. This was a retrospective observational study performed in the emergency department (ED) of a tertiary university hospital visited by 43,000 patients annually. Approximately 200 of those patients present with out-of-hospital cardiac arrest (OHCA). This study was approved by the Institutional Review Board of Wonju Severance Christian Hospital, Yonsei University (IRB No. CR320112).

2.2. Participants. From January 2015 to December 2019, all patients with OHCA who visited the ED were included. The total number of enrolled patients was 1068. All patients underwent treatment according to the current ACLS guidelines [21]. The exclusion criteria were (1) age under 18 years, (2) traumatic cardiac arrest, (3) lack of laboratory study, and (4) insufficient data fidelity in the enrollment chart. We excluded 134 patients with no data on laboratory studies, 177 patients with traumatic cardiac arrest, and 11 patients aged $<18$ years. Eight patients were excluded due to a medical history of leukemia, myelodysplastic syndrome, and myelofibrosis. Finally, 739 patients were enrolled in the study (Figure 1).
2.3. Study Variables. The following clinical and laboratory parameters were obtained: age, sex, total cardiopulmonary resuscitation $(\mathrm{CPR})$ time, neutrophil count, lymphocyte count, platelet count, PLR, NLR, and DNI. The timing of blood sampling was taken during ACLS. Regarding laboratory data, the serum white blood cell, neutrophil, and lymphocyte counts, the platelet count, and the PLR, NLR, and DNI were checked as part of the CBC (ADVIA 2120i Hematology system; Siemens Healthcare Diagnostics, Deerfield, IL, USA). C-reactive protein (Dimension Vista 1500; Siemens Medical Solutions, USA, Inc., Malvern, PA, USA) was used to predict inflammation and infection in the ED. The DNI is calculated based on the number of immature granulocytes using parameters of ADVIA 2120i, as follows: $\mathrm{DNI}=[\%$ neutrophil $+\%$ eosinophil $]-\% \mathrm{PMN}[11]$.

2.4. Study Endpoints. The primary outcome was the ROSC after ACLS. Secondary outcomes were divided into one patient group, including deaths after ROSC ( ${ }^{*}$ including death in the ED; death in the ICU) and survival to discharge (including ${ }^{*}$ home discharge and nursing care discharge) among the patients with ROSC. However, patients were excluded when they were referred to other institutions or were hospitalized after ROSC but could not receive postcardiac arrest care because they were transferred to other institutions within the ICU.

Neurological prognosis was considered as a tertiary outcome in patients who survived to discharge. Neurological prognosis was graded using cerebral performance categories (CPCs). This was measured at the time of hospital discharge and was assessed for outcome. Good neurological outcome was defined as CPCs 1-2, and poor neurological outcome was defined as CPCs 3-5 [22].

2.5. Statistical Analysis. Continuous variables were expressed as mean $\pm \mathrm{SD}$ or median (interquartile range), as appropriate, and categorical variables as counts and percentages (\%). Equal distribution was tested using the Shapiro-Wilk test. Depending on normality, categorical data were analyzed using Fisher's exact or Pearson's chi-square test, while an unpaired $t$-test or Mann-Whitney $U$ test was used to analyze continuous data. Multivariable analysis was performed using a logistic regression model to identify independent predictors of outcome. All $p$ values $<0.05$ were considered statistically significant, and the analysis was performed using SPSS ver. 23 (IBM, Armonk, NY, USA) and MedCalc Statistical Software version 17.5.3 (MedCalc Software, Ostend, Belgium).

\section{Results}

3.1. Baseline Characteristics of the Patients. From January 1, 2015, to December 31, 2019, patients underwent CPR in the hospital's ED. The median age of the enrolled 739 patients was 72 years (range: 19-98 years), and 444 (60.1\%) were male. The median total CPR time was 42 minutes (range: 1-194 minutes), the median C-reactive protein level was 0.95 (range: $0.02-45.60$ ), the median DNI was 0\% (range: 


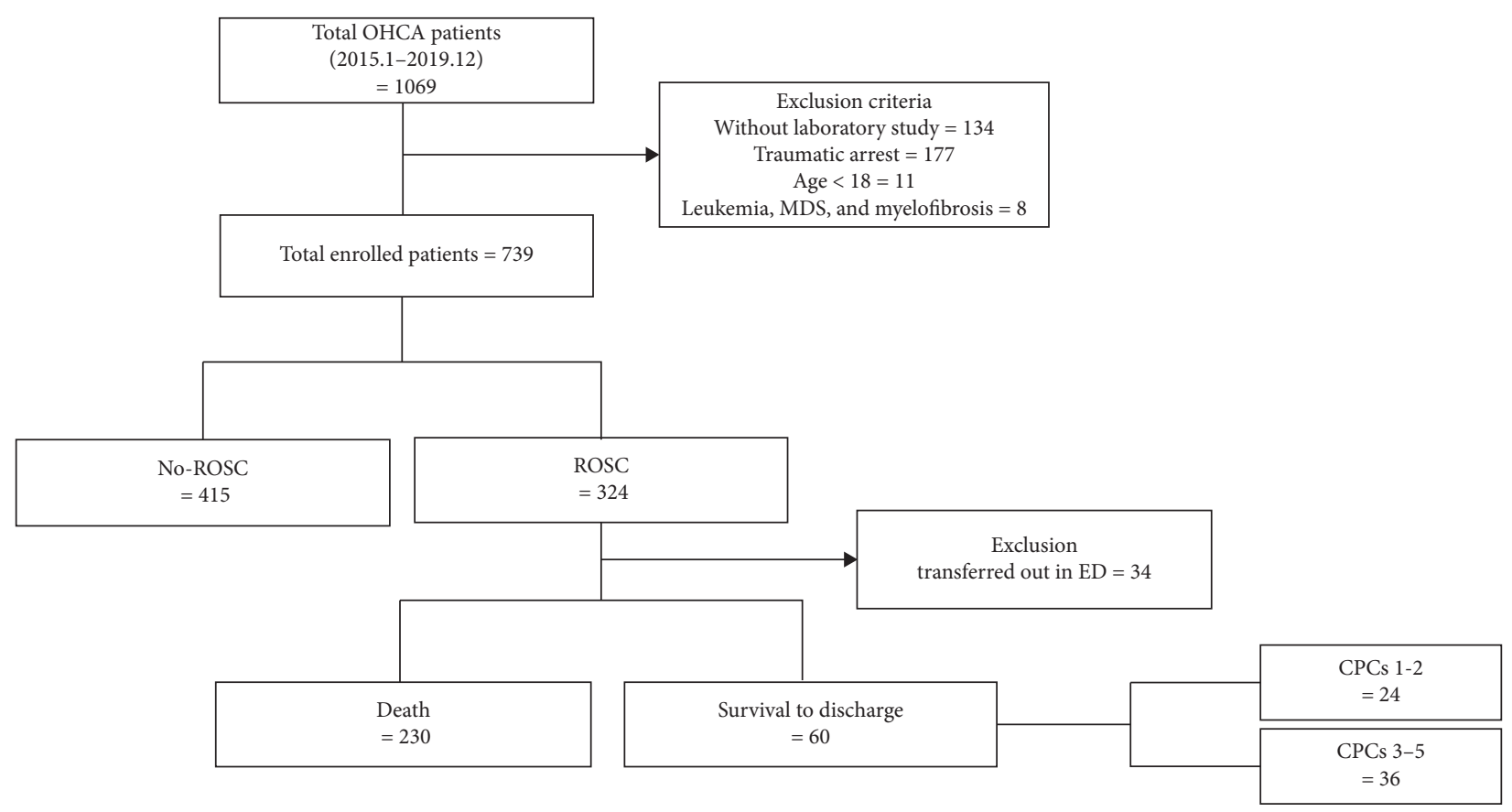

FIgURE 1: Enrollment chart.

$0 \%-57.6 \%$ ), the median neutrophil count was $5.43 \times 10^{9} / \mathrm{L}$ (range: $0.06 \times 10^{9} / \mathrm{L}-43.67 \times 10^{9} / \mathrm{L}$ ), the median lymphocyte count was $4.53 \times 10^{9} / \mathrm{L}$ (range: $0.19 \times 10^{9} / \mathrm{L}-19.82 \times 10^{9} / \mathrm{L}$ ), and the median platelet count was $178 \times 10^{9} / \mathrm{L}$ (range: $5 \times 10^{9} / \mathrm{L}-890 \times 10^{9} / \mathrm{L}$ ). The median PLR was 38.24 (range: 1.94-535.29), while the median NLR was 1.22 (range: 0.04-24.60; Table 1).

3.2. Outcome Assessment. Of the 739 patients analyzed during the study period, 324 had ROSC after ACLS and 415 did not. The median age was 68 years (range: $20-96$ years) in the ROSC group and 75 years (range: $19-98$ years) in the NoROSC group, showing a significant difference. The total CPR time was 52 minutes (range: 2-194 minutes) in the NoROSC group and 31 minutes (range: 1-134 minutes) in the ROSC group. DNI showed no significant difference between the two groups. The PLR value of the ROSC group was 42.41 (range: 4.21-508.7), which was significantly higher than that of the No-ROSC group (34.57; range: 1.94-535.29). The NLR in the ROSC group was 1.38 (range: $-0.09-24.60$ ), which was significantly higher than that in the No-ROSC group (1.08; range: $0.04-21.48$; Table 1 ).

During the study period, 60 patients with ROSC had survival to discharge, while 230 died in the ED or ICU. Thirty-four patients were transferred from the ED to another institution and were excluded from the secondary outcome assessment. The survival to discharge group was younger than the nonsurvival group. In addition, the survival to discharge group underwent a shorter CPR time.

The C-reactive protein level of the survival to discharge group was $0.40 \mathrm{mg} / \mathrm{dL}$ (range: $0.29 \mathrm{mg} / \mathrm{dL}-21.40 \mathrm{mg} / \mathrm{dL}$ ), which was lower than that of the nonsurvival group. The DNI of the survival to discharge group was $0.00 \%$ (range:
$0.0 \%-40.9 \%)$, which was significantly lower than that of the nonsurvival group (0.95\%; range: $0.00 \%-56.20 \%$; Table 1).

The neurological outcomes were evaluated in the 60 patients with survival to discharge. Twenty-four of them had good neurological outcomes, corresponding to CPCs $1-2$, while 36 had poor neurological outcomes corresponding to CPCs 3-5. There was a difference in age between the two groups, and there was a significant difference in total CPR time. The PLR was higher in the group with good neurological outcomes than in the group with poor neurological outcomes, but the difference was not significant (Table 1).

3.3. Predictive Value of Outcomes in Patients with SCA. Univariate and multivariate logistic regression analyses were performed for the first, second, and tertiary outcomes. The predictors of the primary outcome (ROSC) were age and total CPR time, with odds ratios (ORs) of 0.975 (95\% confidence interval (CI): 0.964-0.986) and 0.944 (95\% CI: 0.935-0.954; Table 2), respectively.

Age, total CPR time, and DNI were significant predictors of the secondary outcome (survival to discharge). The OR of age was 0.958 (95\% CI: 0.938-0.978), while that of DNI was 0.924 (95\% CI: 0.859-0.995). The OR of total CPR time was 0.973 (95\% CI: 0.954-0.992). Other values did not differ significantly (Table 3 ).

Univariate and multivariate logistic regression analyses on the tertiary outcome (good neurological outcome) showed that age and total CPR time were significant predictors. The OR of age was 0.950 (95\% CI: 0.910-0.991), while that of total CPR time was 0.953 (95\% CI: 0.912-0.997; Table 4). 


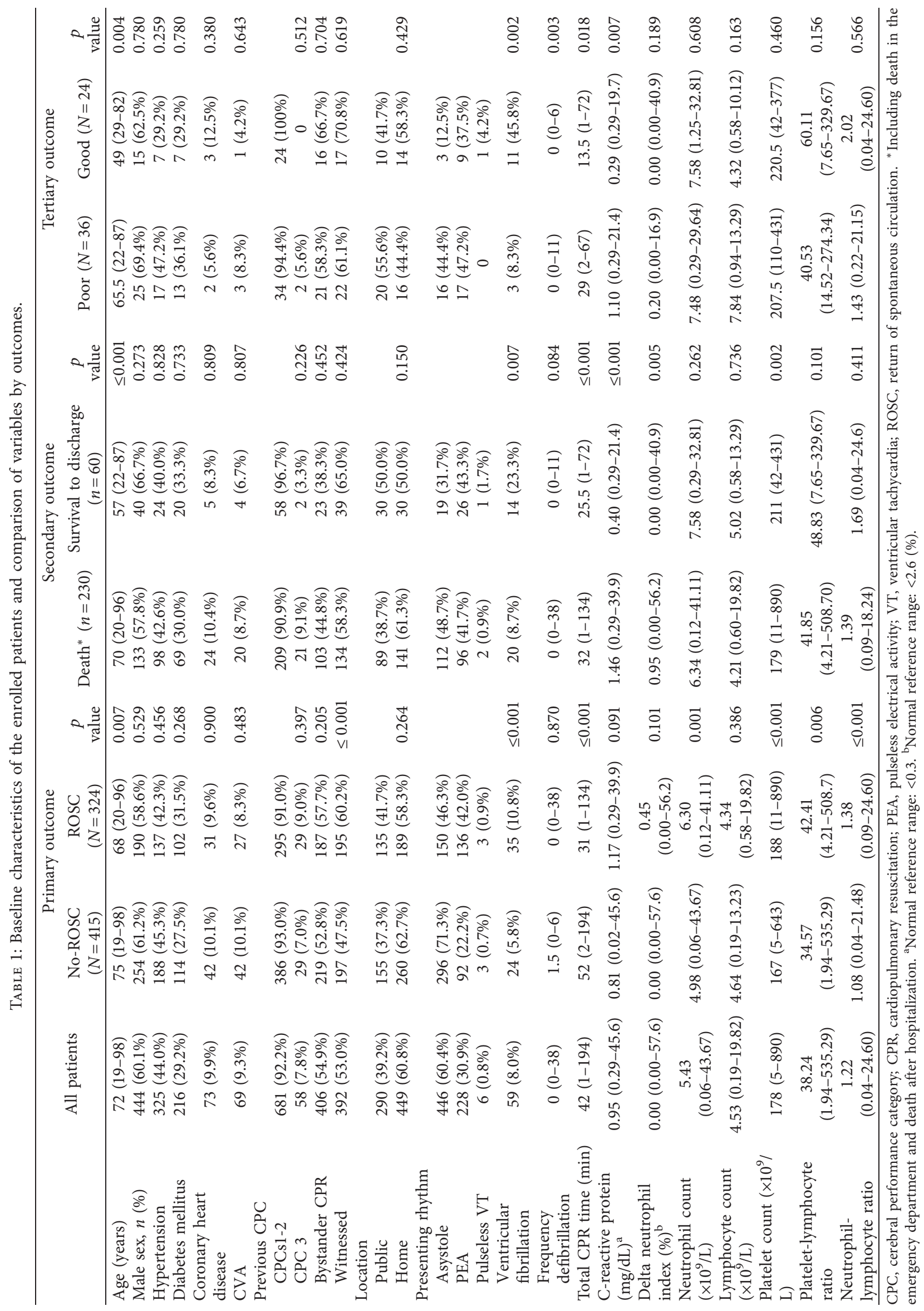


TABLE 2: The predictive factors for the primary outcome (ROSC).

\begin{tabular}{lccccc}
\hline \multirow{2}{*}{ Variable } & \multicolumn{3}{c}{ Univariate logistic regression } & \multicolumn{3}{c}{ Multivariate logistic regression } \\
& OR & $95 \%$ CI & $p$ value & OR & 95\% CI \\
\hline Age & 0.998 & $0.979-0.997$ & 0.013 & 0.975 & $0.964-0.986$ \\
Male sex, $n(\%)$ & 0.832 & $0.668-1.209$ & 0.116 & 0.926 & $0.647-1.324$ \\
Total CPR time & 0.947 & $0.938-0.956$ & $\leq 0.001$ & 0.944 & $0.935-0.954$ \\
CRP & 1.002 & $0.984-1.022$ & 0.803 & & \\
DNI & 1.007 & $0.989-1.025$ & 0.461 & & $\leq 0.001$ \\
PLR & 1.004 & $1.001-1.007$ & 0.004 & 1.003 & $0.998-1.007$ \\
NLR & 1.116 & $1.050-1.185$ & $\leq 0.001$ & 1.004 & $0.916-1.100$ \\
\hline
\end{tabular}

CI, confidence interval; CPR, cardiopulmonary resuscitation; DNI, delta neutrophil index; PLR, platelet-lymphocyte ratio; NLR, neutrophil-lymphocyte ratio.

TABLE 3: The predictive factors for the secondary outcome (survival to discharge).

\begin{tabular}{lccccr}
\hline \multirow{2}{*}{ Variable } & \multicolumn{3}{c}{ Univariate logistic regression } & \multicolumn{2}{c}{ Multivariate logistic regression } \\
& OR & $95 \%$ CI & $p$ value & OR & $95 \%$ CI \\
\hline Age & 0.960 & $0.941-0.978$ & $\leq 0.001$ & 0.958 & $0.938-0.978$ \\
Male sex, $n$ (\%) & 1.458 & $0.803-2.650$ & 0.215 & & $\leq 0.001$ \\
Total CPR time & 0.971 & $0.954-0.988$ & $\leq 0.001$ & 0.973 & $0.954-0.992$ \\
CRP & 0.942 & $0.894-0.994$ & 0.028 & 0.984 & $0.931-1.039$ \\
DNI & 0.926 & $0.869-0.987$ & 0.018 & 0.924 & $0.859-0.995$ \\
PLR & 1.005 & $1.001-1.010$ & 0.012 & 1.006 & $0.999-1.013$ \\
NLR & 1.086 & $1.010-1.168$ & 0.027 & 1.014 & 0.006 \\
\hline
\end{tabular}

$\mathrm{CI}$, confidence interval; CPR, cardiopulmonary resuscitation; DNI, delta neutrophil index; PLR, platelet-lymphocyte ratio; NLR, neutrophil-lymphocyte ratio.

TABLE 4: The predictive factors for the tertiary outcome (good neurological outcome).

\begin{tabular}{lccccc}
\hline \multirow{2}{*}{ Variable } & \multicolumn{3}{c}{ Univariate logistic regression } & \multicolumn{2}{c}{ Multivariate logistic regression } \\
& OR & $95 \%$ CI & $p$ value & OR & $95 \%$ CI \\
\hline Age & 0.951 & $0.916-0.988$ & 0.011 & 0.950 & $0.910-0.991$ \\
Male sex, $n(\%)$ & 0.818 & $0.247-2.179$ & 0.655 & & 0.018 \\
Total CPR time & 0.960 & $0.926-0.996$ & 0.031 & 0.953 & $0.912-0.997$ \\
CRP & 0.895 & $0.776-1.031$ & 0.125 & 0.887 & $0.762-1.033$ \\
DNI & 1.020 & $0.933-1.114$ & 0.664 & & 0.035 \\
PLR & 1.006 & $0.999-1.013$ & 0.099 & 1.004 & $0.996-1.012$ \\
NLR & 1.052 & $0.950-1.165$ & 0.332 & & 0.318 \\
\hline
\end{tabular}

CI, confidence interval; CPR, cardiopulmonary resuscitation; DNI, delta neutrophil index; PLR, platelet-lymphocyte ratio; NLR, neutrophil-lymphocyte ratio.

\section{Discussion}

In this study, we investigated the differences in PLR, NLR, and DNI according to outcomes in patients with cardiac arrest. The study was conducted in a relatively large cohort, and the novel marker PLR is meaningful as a study that first suggested an association with cardiac arrest outcome.

According to previous studies, the following biomarkers predict outcome after cardiac arrest and ROSC: interleukin-6, high-sensitivity C-reactive protein, serum neuron-specific enolase concentration, and S-100 protein [23]. In one study, the risk of death in patients with in-hospital cardiac arrest (IHCA) increased when the cut-off value of NLR was higher than 4.5 [24]. In another study, DNI values higher than $3.3 \%$ at 24 hours significantly predicted 30-day mortality in patients with pediatric cardiac arrest [25]. In a serial follow-up of patients with $\mathrm{OHCA}, \mathrm{DNI}$ at 3 days could be used to assess mortality and neurological outcomes [6].

PLR is a relatively novel marker that has not been investigated in patients with cardiac arrest. In cardiovascular disease, cerebral vascular disease, malignancy, and sepsis, lower PLR values have been associated with better outcomes $[10,26,27]$. However, in the present study, the PLR was higher in patients with ROSC, survival to discharge, and good neurological outcomes than in those without. An important pathophysiology underlying the systemic ische$\mathrm{mia} /$ reperfusion response is disseminated intravascular coagulation in SCA, which leads to microcirculation disorders and thrombocytopenia [17]. But, multivariate logistic regression showed no significant difference in this regard, but the results were nonetheless contrary to those in other diseases. One animal experiment reported that hypoxia $(0.5 \mathrm{~atm})$ reduced the platelet count [28]. However, in other studies, long-term hypoxia reduced platelet count (thrombocytopenia), while short-term hypoxia increased it [29].

Neutrophil survival in vitro can be prolonged by hypoxia where the cells' capacity to generate ROS [30]. Areas of infection and inflammation are hypoxic, so neutrophils operating in such environments are subject to functional modulation by hypoxia [31]. Neutrophils react most rapidly at the onset of 
inflammation, such as in cardiac arrest, and move to the damaged area, causing an acute inflammatory reaction. Therefore, if the initial arrest time is fast, the ROSC rate is also high, and the neutrophil count increases. The faster the arrest detection time from the scene time is, the higher the ROSC ratio is likely to be [32], so the explanatory power of our study can be carried out.

In previous studies, low DNI has been associated with increased survival to discharge $[6,25]$. In the present study, the DNI during initial ACLS was lower in the survival to discharge group than in the short-term mortality group, and the survival to discharge group had a lower DNI than the nonsurvival to discharge group. This corroborates previous studies, which have shown an increased risk of infection and organ failure when immunity and coagulation processes are activated after cardiac arrest, ischemia, and reperfusion. Cytokines, soluble receptors, and endotoxins rise in the early stages, causing interaction between neutrophils and endothelium, as well as an increase in immature granulocytes and consequently in DNI [6].

4.1. Limitation. In this study, there was likely a selection bias because only single-center, retrospective data were used. In addition, there may have been confounding factors, such as cases in the No-ROSC group in which resuscitation was stopped by a caregiver, as well as medically futile situations such as aortic dissection and terminal cancer. Future studies should take these factors into account when designing their inclusion criteria. In addition, research should be conducted using serial follow-up laboratory data.

\section{Conclusion}

In our cohort of patients with SCA, PLR and NLR were associated with ROSC, but among the three blood cell markers, only DNI was associated with survival to discharge. However, unlike other diseases, PLR and NLR were less valuable as predictors.

\section{Data Availability}

The patient agreed to this data collection and study but did not consent to data sharing.

\section{Conflicts of Interest}

The authors declare that there are no conflicts of interest.

\section{References}

[1] Y. Moriwaki, Y. Tahara, T. Kosuge, and N. Suzuki, "Etiology of out-of-hospital cardiac arrest diagnosed via detailed examinations including perimortem computed tomography," Journal of Emergencies, Trauma, and Shock, vol. 6, no. 2, pp. 87-94, 2013.

[2] Centers for Disease Control Prevention, "State-specific mortality from sudden cardiac death-United States, 1999," MMWR Morbidity and Mortality Weekly Report, vol. 51, no. 6, pp. 123-126, 2002.
[3] M. E. Kleinman, E. E. Brennan, Z. D. Goldberger et al., "Part 5: adult basic life support and cardiopulmonary resuscitation quality: 2015 American Heart Association guidelines update for cardiopulmonary resuscitation and emergency cardiovascular care," Circulation, vol. 132, no. 18, pp. S414-S435, 2015.

[4] A. Mangla, M. R. Daya, and S. Gupta, "Post-resuscitation care for survivors of cardiac arrest," Indian Heart Journal, vol. 66, no. 1, pp. S105-S112, 2014.

[5] J. Soar, J. P. Nolan, B. W. Böttiger et al., "European resuscitation council guidelines for resuscitation 2015," Resuscitation, vol. 95, pp. 100-147, 2015.

[6] H. Y. Yune, S. P. Chung, Y. S. Park et al., "Delta neutrophil index as a promising prognostic marker in out of hospital cardiac arrest," PLoS One, vol. 10, no. 3, Article ID e0120677, 2015.

[7] S. P. Chung, H. Y. Yune, Y. S. Park et al., "Usefulness of mean platelet volume as a marker for clinical outcomes after out-ofhospital cardiac arrest: a retrospective cohort study," Journal of Thrombosis and Haemostasis, vol. 14, no. 10, pp. 2036-2044, 2016.

[8] A. Cotoia, F. Franchi, C. De Fazio, J. L. Vincent, J. Creteur, and F. S. Taccone, "Platelet indices and outcome after cardiac arrest," BMC Emergency Medicine, vol. 18, no. 1, p. 31, 2018.

[9] A. Papa, M. Emdin, C. Passino, C. Michelassi, D. Battaglia, and F. Cocci, "Predictive value of elevated neutrophil-lymphocyte ratio on cardiac mortality in patients with stable coronary artery disease," Clinica Chimica Acta, vol. 395, no. 12, pp. 27-31, 2008.

[10] S. Balta and C. Ozturk, "The platelet-lymphocyte ratio: a simple, inexpensive and rapid prognostic marker for cardiovascular events," Platelets, vol. 26, no. 7, pp. 680-681, 2015.

[11] C. H. Nahm, J. W. Choi, and J. Lee, "Delta neutrophil index in automated immature granulocyte counts for assessing disease severity of patients with sepsis," Annals of Clinical and Laboratory Science, vol. 38, no. 3, pp. 241-246, 2008.

[12] S. Balta, S. Demirkol, and U. Kucuk, "The platelet lymphocyte ratio may be useful inflammatory indicator in clinical practice," Hemodialysis International. International Symposium on Home Hemodialysis, vol. 17, no. 4, pp. 668-669, 2013.

[13] T. Angkananard, T. Anothaisintawee, M. McEvoy, J. Attia, and A. Thakkinstian, "Neutrophil lymphocyte ratio and cardiovascular disease risk: a systematic review and metaanalysis," Biomed Research International, vol. 2018, Article ID 2703518, 11 pages, 2018.

[14] T. Y. Kim, S. J. Kim, Y. S. Kim et al., "Delta neutrophil index as an early predictive marker of severe acute pancreatitis in the emergency department," United European Gastroenterology Journal, vol. 7, no. 4, pp. 488-495, 2019.

[15] B. H. Park, Y. A. Kang, M. S. Park et al., "Delta neutrophil index as an early marker of disease severity in critically ill patients with sepsis," BMC Infectious Diseases, vol. 11, p. 299, 2011.

[16] N. Mongardon, F. Dumas, S. Ricome et al., "Postcardiac arrest syndrome: from immediate resuscitation to long-term outcome," Annals of Intensive Care, vol. 1, no. 1, p. 45, 2011.

[17] T. Wada, "Coagulofibrinolytic changes in patients with postcardiac arrest syndrome," Frontiers in Medicine, vol. 4, p. 156, 2017.

[18] P. Villois, D. Grimaldi, S. Spadaro et al., "Lymphopaenia in cardiac arrest patients," Annals of Intensive Care, vol. 7, no. 1, p. 85, 2017.

[19] J. L. Weidman, D. C. Shook, and J. N. Hilberath, "Cardiac resuscitation and coagulation," Anesthesiology, vol. 120, no. 4, pp. 1009-1014, 2014. 
[20] R. W. Neumar, J. P. Nolan, C. Adrie et al., "Post-cardiac arrest syndrome: epidemiology, pathophysiology, treatment, and prognostication. A consensus statement from the International Liaison Committee on Resuscitation (American Heart Association, Australian and New Zealand Council on Resuscitation, European Resuscitation Council, Heart and Stroke Foundation of Canada, InterAmerican Heart Foundation, Resuscitation Council of Asia, and the Resuscitation Council of Southern Africa); the American Heart Association Emergency Cardiovascular Care Committee; the Council on Cardiovascular Surgery and Anesthesia; the Council on Cardiopulmonary, Perioperative, and Critical Care; the Council on Clinical Cardiology; and the Stroke Council," Circulation, vol. 118, no. 23, pp. 2452-2483, 2008.

[21] M. S. Link, L. C. Berkow, P. J. Kudenchuk et al., "Part 7: adult advanced cardiovascular life support: 2015 American Heart Association guidelines update for cardiopulmonary resuscitation and emergency cardiovascular care," Circulation, vol. 132, no. 18, pp. S444-S464, 2015.

[22] E. Edgren, U. Hedstrand, S. Kelsey, K. Sutton-Tyrrell, and P. Safar, "Assessment of neurological prognosis in comatose survivors of cardiac arrest," The Lancet, vol. 343, no. 8905, pp. 1055-1059, 1994.

[23] M. Karapetkova, M. A. Koenig, and X. Jia, "Early prognostication markers in cardiac arrest patients treated with hypothermia," European Journal of Neurology, vol. 23, no. 3, pp. 476-488, 2016.

[24] V. H. Patel, P. Vendittelli, R. Garg et al., "Neutrophil-lymphocyte ratio: a prognostic tool in patients with in-hospital cardiac arrest," World Journal of Critical Care Medicine, vol. 8, no. 2, pp. 9-17, 2019.

[25] S. H. Yoon, E. J. Lee, J. Lee, M. K. Kim, and J. G. Ahn, "Prognostic value of the delta neutrophil index in pediatric cardiac arrest," Scientific Reports, vol. 10, no. 1, p. 3497, 2020.

[26] T. Gary, M. Pichler, K. Belaj et al., "Platelet-to-lymphocyte ratio: a novel marker for critical limb ischemia in peripheral arterial occlusive disease patients," PLoS One, vol. 8, no. 7, Article ID e67688, 2013.

[27] Y. Shen, X. Huang, and W. Zhang, "Platelet-to-lymphocyte ratio as a prognostic predictor of mortality for sepsis: interaction effect with disease severity-a retrospective study," BMJ Open, vol. 9, no. 1, Article ID e022896, 2019.

[28] J. W. Birks, L. W. Klassen, and C. W. Gurney, "Hypoxiainduced thrombocytopenia in mice," The Journal of Laboratory and Clinical Medicine, vol. 86, no. 2, pp. 230-238, 1975.

[29] T. McDonald, M. Cottrell, and R. Clift, "Effects of short-term hypoxia on platelet counts of mice," Blood, vol. 51, no. 1, pp. $165-175,1978$.

[30] S. Hannah, K. Mecklenburgh, I. Rahman et al., "Hypoxia prolongs neutrophil survival in vitro," FEBS Letters, vol. 372, no. 2-3, pp. 233-237, 1995.

[31] K. M. Lodge, A. S. Cowburn, W. Li, and A. M. Condliffe, "The impact of hypoxia on neutrophil degranulation and consequences for the host," International Journal of Molecular Sciences, vol. 21, no. 4, p. 1183, 2020.

[32] C. Welbourn and N. Efstathiou, "How does the length of cardiopulmonary resuscitation affect brain damage in patients surviving cardiac arrest? A systematic review," Scandinavian Journal of Trauma, Resuscitation and Emergency Medicine, vol. 26, no. 1, p. 77, 2018. 